\title{
GASIFICACIÓN DEL ASERRÍN DE PINO (Pinus arizonica) EN UN REACTOR DE LECHO FLUIDIZADO: ANALISIS DE LAS VARIABLES DEL PROCESO
}

\author{
PINE SAWDUST (Pinus arizonica) GASIFICATION IN A \\ FLUIDIZED BED REACTOR: ANALYSIS OF PROCESS \\ VARIABLES
}

\author{
Martin Enrique Durán-García ${ }^{1, \$}$
}

\begin{abstract}
RESUMEN
La gasificación hidrotérmica del aserrín de pino (Pinus arizonica) es un proceso de degradación termoquímica de sus macromoléculas poliméricas con el fin de producir principalmente hidrógeno y metano como biocombustibles. Este proceso puede ocurrir en un reactor de lecho fluidizado que permite un apropiado contacto gas-sólido, el adecuado manejo de la temperatura y una mayor velocidad de reacción. El propósito es modelar y simular las variables del proceso donde se gasifica esta biomasa forestal a partir del respectivo análisis de sensibilidad. Se obtiene que la conversión de reacción de gasificación del aserrín de pino crece a una temperatura mayor a los $900{ }^{\circ} \mathrm{C}$, presentando un comportamiento que tiende a ser isotérmico, por lo que se sugiere un proceso energéticamente autosostenible. A su vez, la reacción mejoró para una alimentación de aserrín de pino con un diámetro óptimo de tamaño de partícula de $0,65 \mathrm{~mm}$; se ve favorecida para bajas relaciones de vapor de agua/ biomasa alimentada alcanzando el óptimo en 2,375; y a bajas relaciones aire/biomasa con un valor óptimo de 0,289 . Se evidencia que este proceso constituye una vía de aprovechamiento energético a partir de la conversión termoquímica de estos desechos y residuos forestales.
\end{abstract}

Palabras Claves: Biomasa forestal, modelación, proceso termoquímico, reactor químico, simulación.

\begin{abstract}
The hydrothermal gasification of sawdust pine (Pinus arizonica) is a process of thermochemical degradation of polymeric macromolecules in order to produce mainly hydrogen and methane as biofuels. This process can occur in a fluidized bed reactor which allows an appropriate gas-solid, proper temperature management and a faster reaction. The purpose is to model and simulate process variables where this forest biomass is gasified from the respective sensitivity analysis. It is obtained that the conversion of gasification reaction of pine sawdust increases at temperature higher than 900

\footnotetext{
${ }^{1}$ Departamento de Tecnología Industrial, Universidad Simón Bolívar, Caracas, Venezuela.

•Autor para correspondencia: martinduran@usb.ve

Recibido: 03.10.2015 Aceptado: 27.07.2016
} 
${ }^{\circ} \mathrm{C}$, showing a behavior that tends to be isothermal, suggesting an energetically self-sustaining process. In turn, it improved reaction for pine sawdust feed with an optimum particle size diameter of $0,65 \mathrm{~mm}$; it is favored for low ratios steam / biomass fed in reaching the optimal 2,375; and at low air / biomass relationship with an optimum value of 0,289 . It is evident that this process is a way of harnessing energy from thermochemical conversion of these wastes and forestry residues.

Keywords: Chemical reactor, forest biomass, modeling, simulation, thermochemical process.

\section{INTRODUCCIÓN}

El proceso de gasificación hidrotérmica representa una vía de aprovechamiento energético de residuos forestales, mediante su transformación termoquímica (degradación de macromoléculas poliméricas) en un gas combustible de gran poder calorífico por su alto contenido en hidrógeno e hidrocarburos ligeros (Antal, et al. 2000, Guo et al. 2007). Este proceso se realiza principalmente en unidades de reacción química como los mencionados reactores de lecho fijo, fluidizado y burbujeante, por lo que resulta importante el diseño de estos equipos donde ocurre la gasificación de la biomasa forestal como el aserrín de pino (Pinus arizonica).

Específicamente se modelará un reactor lecho fluidizado como unidad de reacción química encargada de la conversión de esta biomasa forestal, con el fin de verificar el desempeño del mismo ante el cambio de las variables de operación como porcentaje en peso de los gases producidos, la temperatura, relaciones de alimentación de biomasa con el agente gasificante, y el tamaño de partícula de la biomasa alimentada (Antal, et al. 2000, Lee et al. 2002a, Lee et al. 2002b, Ramakers et al. 2004, Guo et al. 2007, Fang et al. 2008, Soto y Nuñez 2008, Beraldo y Balzamo 2009, Kumar et al. 2009).

La gasificación hidrotérmica de la biomasa constituye un conjunto de reacciones termoquímicas que se producen en un ambiente pobre en oxígeno y que da como resultado la trasformación de un sólido, conformado por principalmente por la celulosa, lignina, hemicelulosa y extraíbles que son degradadas por estos termoquímicos, que se convierte en una mezcla de gases combustibles principalmente constituida por metano, hidrógeno, dióxido de carbono, monóxido de carbono, con su correspondiente poder calorífico y susceptibles de ser utilizados en sistemas industriales constituidos principalmente por calderas, turbinas, motores o máquinas de combustión, entre otros, tras ser debidamente acondicionados (Lee et al. 1998, Lee et al. 2002a, Lu et al. 2006, Lu et al. 2007, Lu et al. 2008, Guo et al. 2007, Fang et al. 2008, Kumar et al. 2009, Durán-García 2014).

\section{Reactor de lecho fluidizado}

Los reactores pueden diferir en función de las características del tipo de alimentación de la biomasa residual a gasificar. Los gasificadores habituales son de lecho fijo y a su vez pueden ser de flujo de gas ascendente o descendente. También se han desarrollado gasificadores de lecho fluidizado con ciertas ventajas respecto a los anteriores, ya que presentan mayores velocidades de reacción y eficiencias de conversión superiores siendo uno de los sistemas más favorables (Kumar et al. 2009).

El reactor de lecho fluidizado es aquel donde el agente gasificante mantiene en suspensión a un inerte y a la biomasa, hasta que las partículas de éste se gasifican y se convierten en cenizas volátiles las cuales son arrastradas por la corriente correspondiente. Se observa el ascenso lento de las burbujas pequeñas, que pasa a ser más rápido en las grandes, pudiendo una serie de burbujas formar por coalescencia burbujas de mayor tamaño (Guo et al. 2007, Fang et al. 2008, Nikoo and Mahinpey 2008, Durán-García 2014).

Las ventajas de gasificar el aserrín de pino en este reactor se fundamenta en el buen control de temperatura, los altos flujos de reacción, los niveles moderados de alquitrán generado, el apropiado contacto gas - sólido, su operación con carga parcial, su fácil construcción y operación, los elevados niveles de conversión, el comportamiento de las partículas como un fluido permite controlar los procesos fácilmente, el buen mezclado de los sólidos satisface el comportamiento isotermo a través del 
reactor, el mezclado homogéneo de los sólidos genera una inercia térmica que impide cambios bruscos de temperatura y da un amplio margen de seguridad para evacuar el calor en procesos muy exotérmicos y los coeficientes de transferencia de calor y de masa son altos comparados con otros procesos (Kunii and Levenspiel 1991, Guo et al. 2007, Lu et al. 2007, Lu et al. 2008, Fang et al. 2008, Nikoo and Mahinpey 2008, Durán-García 2014).

\section{METODOLOGÍA}

La metodología comprende el modelaje de la gasificación del aserrín de pino (Pinus arizonica Engelm) a través de la formulación del modelo matemático del reactor: modelo cinético, velocidad de reacción, balance de masa, balance de energía, fluidodinámica, geometría y dimensiones del reactor bajo las condiciones de diseño y operación en estudio. Para ello se revisaron los trabajos previos en la literatura y se adaptaron algunas ecuaciones que mejor permitían la descripción del fenómeno de gasificación hidrotérmica de esta biomasa forestal (Nikoo and Mahinpey 2008, Duran-García 2014, Lee et al. 2002a, Guo et al. 2007, Fang et al. 2008, Kumar et al. 2009).

Posteriormente se realizó la simulación del modelo formulado en el software matemático POLIMATH EDUCATIONAL 6.0 CACHE Corporation, con la finalidad de determinar el rango de temperatura más apropiado que permita la mayor producción de los gases combustibles como hidrógeno o metano, verificar el comportamiento isotérmico del reactor y el impacto del tamaño de partícula en el rendimiento del proceso, el efecto de las relaciones de vapor/biomasa alimentada y aire/biomasa para los biocombustibles formados, a partir del correspondiente análisis de sensibilidad.

La correspondiente formulación del modelo matemático del reactor se presenta a continuación:

\section{Modelo cinético}

Las reacciones involucradas en el proceso de gasificación pueden simplificarse en dos reacciones principales:

\section{- Reacción de combustión}

$$
C_{(S)}+0,9 \mathrm{O}_{2(g)} \rightarrow 0,2 \mathrm{CO}_{(g)}+0,8 \mathrm{CO}_{2(g)} \Delta \mathrm{H}_{R X}^{0}=-336,9 \mathrm{Kj} / \mathrm{mol}
$$

- Reacción de gasificación

$$
C_{(S)}+\mathrm{H}_{2} \mathrm{O}_{(g)} \rightarrow \mathrm{CO}_{(g)}+\mathrm{H}_{2(g)} \Delta \mathrm{H}_{R X}^{0}=131,3 \mathrm{Kj} / \mathrm{mol}
$$
(2008)

Para la reacción de combustión, se plantea la ley de velocidad empleada por Nikoo and Mahinpey

$$
r_{1}=k_{1} P_{\mathrm{o}_{2}}(1-x)^{2 / 3} \frac{\rho_{c} \varepsilon_{s} Y_{c}}{P M_{c}}
$$

Ahora bien, expresando la ecuación anterior en función de la conversión del oxígeno expresado en presiones parciales iniciales y finales de oxígeno, ya que el carbono está en fase sólida se obtiene:

$$
\left.r_{1}=k_{1} P_{\mathrm{o}}\left(-\frac{10}{\left(\frac{P_{\mathrm{o}_{2}}}{P} P_{\mathrm{o}_{2}}\right.}\right)\right)^{2 / 3} \frac{\rho_{c} \varepsilon_{s} c}{P M}
$$


Para la reacción de gasificación, se plantea la ley de velocidad propuesta por Kojima et al. (1993) que depende solamente de la presión parcial del agente gasificante, la cual establece:

$$
r_{2}=k_{2}\left(P_{H_{2} \mathrm{O}} \times 10^{3}\right)^{0,41} \frac{\rho_{c} \varepsilon_{s} Y_{c}}{P M_{c}}
$$

\section{Ecuaciones de Balance de Masa}

Las leyes de velocidad expresadas en función de cada especie se plantean a continuación:

$$
\begin{aligned}
& r_{c}=-r_{1}-r_{2} \\
& r_{o_{2}}=-\frac{10}{9} r_{1} \\
& r_{c o}=5 r_{1}+r_{2} \\
& r_{c o_{2}}=\frac{5}{4} r_{1} \\
& r_{H_{2} \mathrm{O}}=-r_{2} \\
& r_{H_{2}}=-r_{2}
\end{aligned}
$$

El modelo fluodinámico del reactor está expresado en función de una distancia z y la geometría tubular del reactor, de donde se propone simularlo como un reactor flujo pistón ideal (ya que no se disponen datos de RTD), por tanto la ecuación de balance de masa en función del volumen total de sistema de reacción queda establecida para cada especie como:

$$
\frac{d F_{i}}{d V}=A_{\text {reactor }} r_{i}
$$

\section{Ecuación de Balance de Energía}

Para éste caso, se simuló el reactor bajo la modalidad de un flujo pistón adiabático donde la ecuación diferencial se expresa en los siguientes términos:

$$
\frac{d T}{d V}=A_{\text {reactor }} \frac{\sum r_{1} \Delta \mathrm{H}_{R X, i}}{\sum F_{i} C_{p i}}
$$

De donde,

$$
\Delta \mathrm{H}_{R X, i}=\Delta \mathrm{H}_{R X, i}{ }^{\circ}+\Delta C_{p}\left(T-T^{o}\right)
$$




\section{Modelo Fluodinámico}

Para ésta simulación se partió del modelo empleado por Nikoo and Mahinpey (2008), el cual plantea que la fracción volumétrica de sólidos varía a lo largo del reactor en función de la distancia (z) de la forma:

$$
\begin{gathered}
\varepsilon_{s}=\left(1-\varepsilon_{f}\right) \exp (-a z) \\
a=\frac{1,8}{U} \\
\varepsilon_{f}=\varepsilon_{b}+\varepsilon_{m f}\left(1-\varepsilon_{b}\right) \\
\varepsilon_{m f}=0,4 \\
B=1+\frac{10,978\left(U-U_{m f}\right)^{0,738} \rho_{s}{ }^{0,376} d p^{1,006}}{U_{m f}{ }^{0,937} \rho_{g}{ }^{0,126}} \\
U_{m f}=\frac{1-\frac{1}{B}}{\rho_{g} d p}\left({\sqrt{1-3,59 x 10^{-5} A r-1}}^{3} \rho_{g}\left(\rho_{s}-\rho_{g}\right) \mathrm{g}\right. \\
\mu^{2}
\end{gathered}
$$

\section{Suposiciones o simplificaciones del modelo}

Las consideraciones planteadas para resolver el algoritmo de solución de este reactor son las siguientes:

- Estado estacionario

- Se desprecia la resistencia a la transferencia de masa y calor en la velocidad de reacción

- El reactor se comporta como un flujo pistón ideal

- El reactor opera adiabáticamente

- No se considera la disposición de cenizas en el reactor

- No se considera caída de presión apreciable a lo largo del reactor

- La fase gaseosa del reactor se comporta como gas ideal 


\section{Geometría del Reactor}

Se emplearon las dimensiones del reactor utilizado experimentalmente y bajo distintos escenarios de simulación por Nikoo and Mahinpey (2008) y Durán-García (2014) las cuales se observan en la Tabla 1.

Tabla 1. Geometría del reactor simulado.

\begin{tabular}{|c|c|}
\hline Parámetro & Valor $(\mathbf{m m})$ \\
\hline Diámetro interno & 60 \\
\hline Longitud & 1400 \\
\hline
\end{tabular}

\section{DISCUSIÓN Y ANÁLISIS DE RESULTADOS}

Los resultados como su discusión se fundamenta en los valores obtenidos en la simulación del proceso: el rango de temperatura, el comportamiento isotérmico del reactor y el impacto del tamaño de partícula en el rendimiento del proceso, el efecto de las relaciones de vapor/biomasa alimentada y aire/ biomasa para los biocombustibles formados, a partir del correspondiente análisis de sensibilidad; los cuales se presentan a través de los siguientes casos.

\section{Caso 1. Variación de la temperatura de alimentación}

Los resultados y análisis de este caso se detallan a partir de las condiciones de operación del reactor que se reflejan en la Tabla 2.

Tabla 2. Condiciones de operación del reactor.

\begin{tabular}{|l|c|}
\hline \multicolumn{1}{|c|}{ Parámetro } & Valor \\
\hline Flujo biomasa $(\mathrm{kg} / \mathrm{h})$ & 0,444 \\
\hline Flujo aire $\left(\mathrm{Nm}^{3} / \mathrm{h}\right)$ & 0,5 \\
\hline Flujo vapor agua $(\mathrm{kg} / \mathrm{h})$ & 1,8 \\
\hline Diámetro de la partícula $(\mathrm{mm})$ & 0,25 \\
\hline
\end{tabular}

La variación de la conversión global del carbono en función de la temperatura se presenta en la Figura 1.

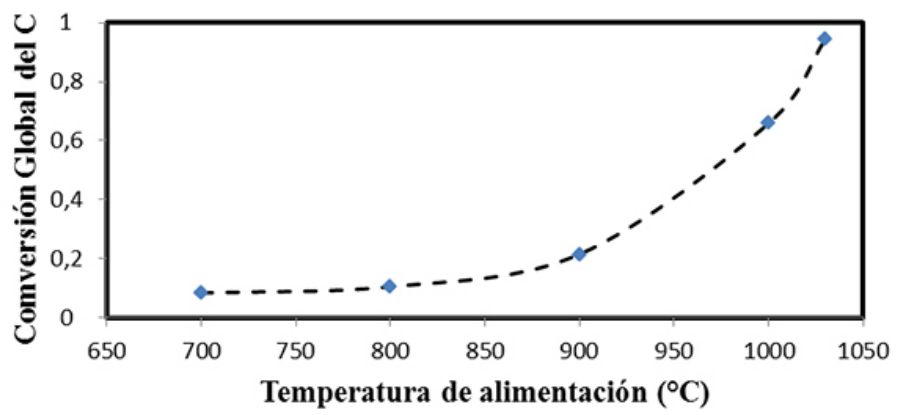

Figura 1. Variación de la conversión global del carbono en función de la temperatura de alimentación de los reactivos. 
Se observa que la conversión global de Carbono aumenta a medida que la temperatura de alimentación se incrementa, evidenciándose un crecimiento promedio de la conversión global del carbono de 0,215 por cada $50^{\circ} \mathrm{C}$, en el rango comprendido entre $900-1000{ }^{\circ} \mathrm{C}$.

La variación de la composición del gas de emisión se presenta en la Figura 2:

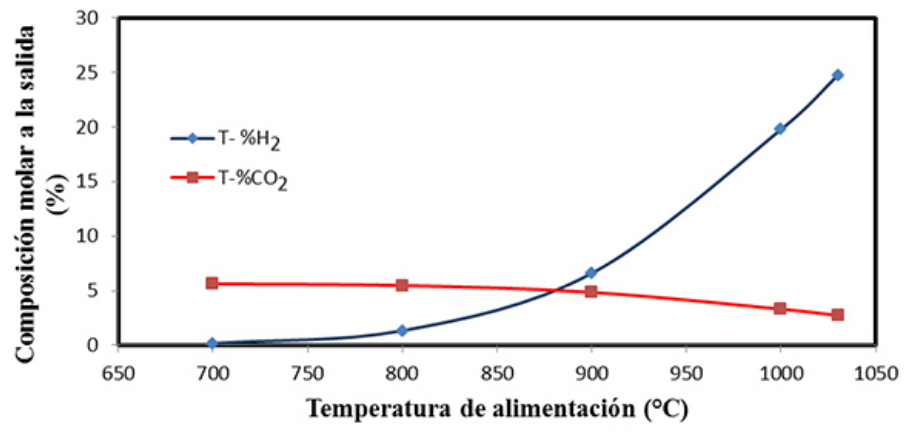

Figura 2. Variación de la composición del hidrógeno y dióxido de carbono en función de la temperatura de alimentación de los reactivos.

Se observa principalmente a bajas temperaturas la presencia de dióxido de carbono, debido a que predomina la reacción de combustión por encima de la reacción de gasificación. A partir de $900{ }^{\circ} \mathrm{C}$ comienza a dominar la reacción de gasificación lo cual repercute en la concentración de hidrógeno en la mezcla de gases resultantes.

En cuanto a la variación de la diferencia de temperatura entre la entrada y salida del reactor se puede apreciar en la Figura 3. Se evidencia que al aumentar la temperatura de alimentación el diferencial de temperatura en el reactor tiende a incrementarse, alcanzando la condición isotérmica a los $918{ }^{\circ} \mathrm{C}$ aproximadamente. De allí hacia valores más altos se observa que el diferencial toma valores positivos, reflejando que el reactor comienza a generar calor.

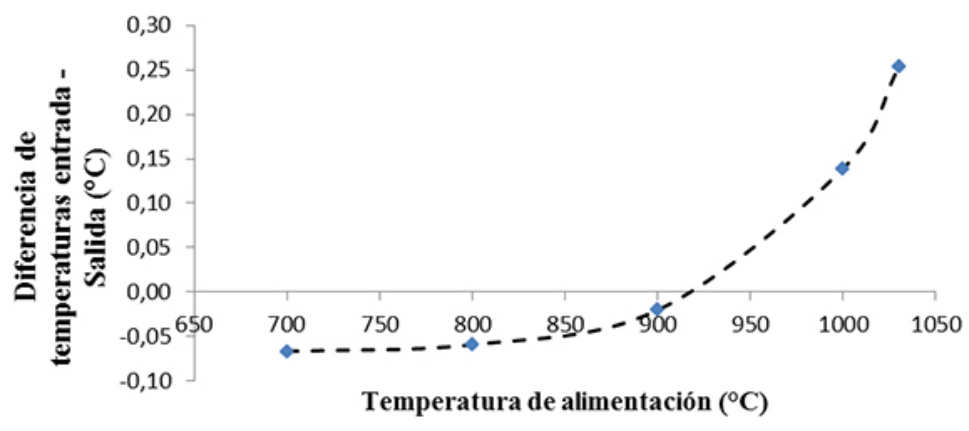

Figura 3. Variación de la diferencia de temperatura en el reactor en función de la temperatura de alimentación de los reactivos.

El balance energético es positivo lo que sugiere que la reacción puede ser autosostenible (exotérmica) a partir de ese nivel de temperatura. No obstante, el diferencial de temperatura observado es realmente bajo, lo cual tiende a un comportamiento isotérmico en términos generales.

La variación del rendimiento obtenido en función de la temperatura de entrada se aprecia en la Figura 4: 


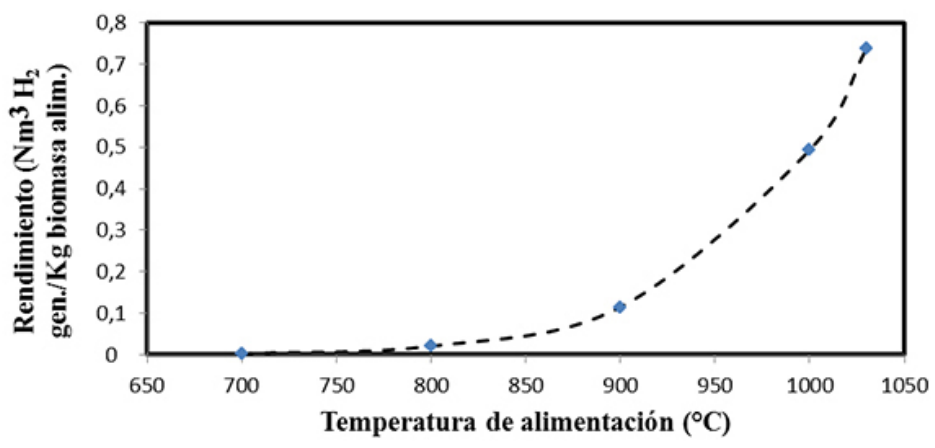

Figura 4. Variación del rendimiento en función de la temperatura de alimentación de los reactivos.

Se evaluó en rendimiento de la reacción en términos de $\mathrm{Nm}^{3}$ de $\mathrm{H}_{2}$ generado por $\mathrm{kg}$ de biomasa alimentado, donde a valores superiores de temperaturas de alimentación se observa un mayor rendimiento, debido a que aumenta la conversión y a su vez se infiere que se debe al aumento en la selectividad de la reacción de gasificación.

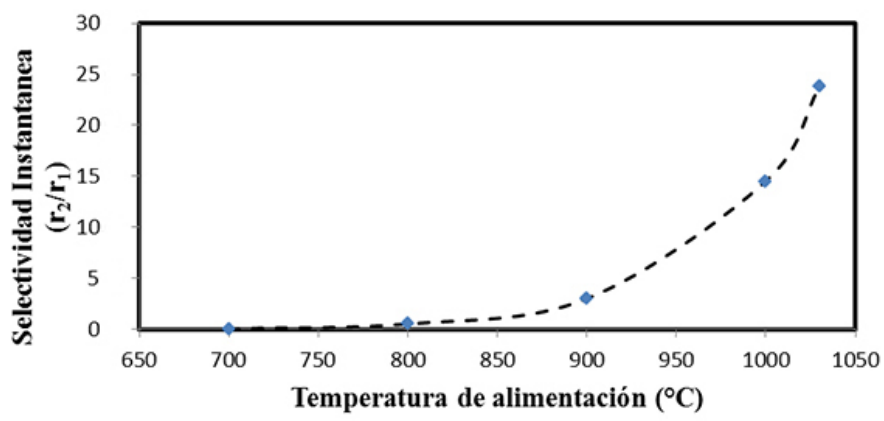

Figura 5. Variación de la selectividad instantánea en función de la temperatura de alimentación de los reactivos.

En cuanto a la variación de la selectividad instantánea (Figura 5), se tiene que a medida que la temperatura de alimentación se incrementa, la selectividad instantánea de la reacción de gasificación en función de la reacción de combustión aumenta, predominando está a partir de $900{ }^{\circ} \mathrm{C}$ en adelante.

\section{Caso 2. Variación del tamaño de la partícula de biomasa alimentada}

En este caso se presentan los resultados y sus análisis a partir de las condiciones de operación del reactor, las cuales se reflejan en la Tabla 3, donde se resalta que la temperatura de operación es la mejor obtenida en la primera parte de los análisis.

Tabla 3. Condiciones de operación del reactor.

\begin{tabular}{|l|c|}
\hline \multicolumn{1}{|c|}{ Parámetro } & Valor \\
\hline Flujo biomasa $(\mathrm{kg} / \mathrm{h})$ & 0,444 \\
\hline Flujo aire $\left(\mathrm{Nm}^{3} / \mathrm{h}\right)$ & 0,5 \\
\hline Flujo vapor agua $(\mathrm{kg} / \mathrm{h})$ & 1,8 \\
\hline Temperatura de entrada $\left({ }^{\circ} \mathrm{C}\right)$ & 900 \\
\hline
\end{tabular}


La variación de la conversión global del carbono se presenta en la Figura 6.

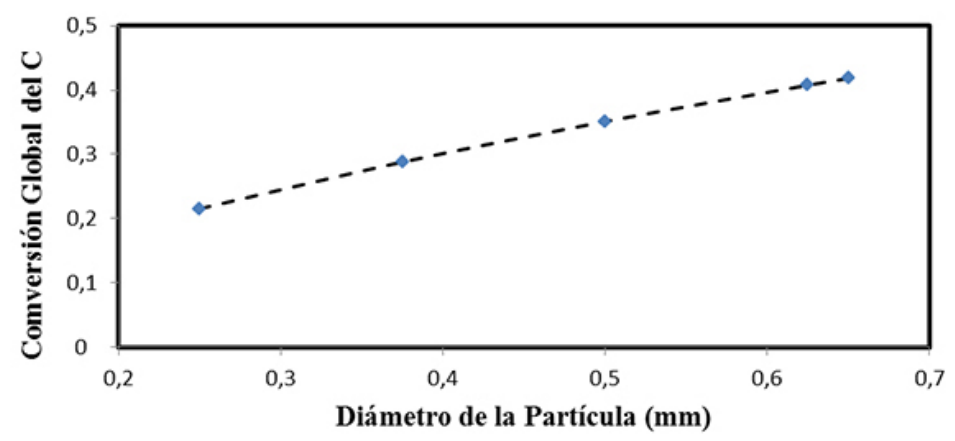

Figura 6. Variación de la conversión global del carbono en función del diámetro de la partícula.

Se observa que para un sistema a temperatura de entrada de reactivos constante, la conversión del carbono se ve beneficiada a mayores tamaños de partícula, donde se puede resaltar que el valor óptimo de $0,65 \mathrm{~mm}$; en el rango entre 0,25 y $0,65 \mathrm{~mm}$.

En la variación de la composición del gas de emisión a medida que se aumenta el diámetro de la partícula en el rango estudiado (Figura 7), se observa que la composición del dióxido de carbono (producto predominante de la primera reacción) tiene una tendencia característica, de tal forma que adquiere un máximo y tiende a descender en los mayores valores de diámetros estudiados. En el caso del hidrogeno, la tendencia es creciente a medida que aumenta el diámetro de la partícula.

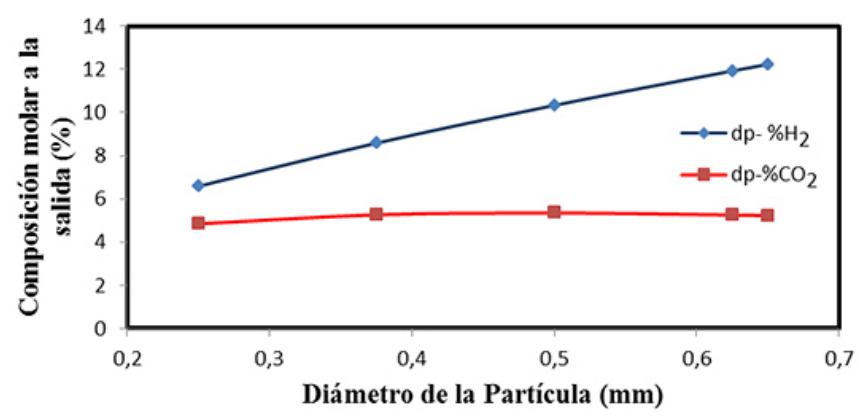

Figura 7. Variación de la composición molar del hidrógeno y dióxido de carbono en función del diámetro de la partícula.

A medida que se aumenta el diámetro de la partícula en el rango estudiado, se observa que la composición de dióxido de carbono (producto predominante de la primera reacción) tiene una tendencia característica, de tal forma que adquiere un máximo y tiende a descender en los mayores valores de diámetros estudiados. Para el caso del hidrogeno, la tendencia es creciente a medida que aumenta el diámetro de la partícula.

La variación de la diferencia de temperatura entre la entrada y salida del reactor se presenta en la Figura 8: 


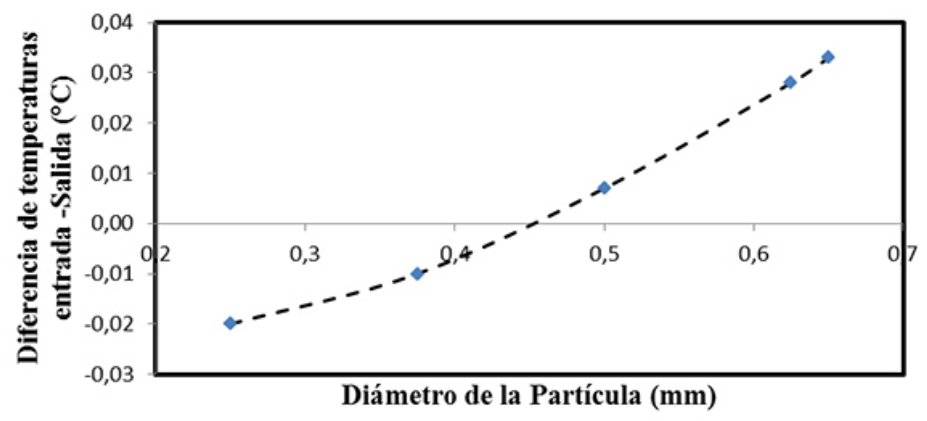

Figura 8. Variación de la diferencia de temperatura en el reactor de en función del diámetro de la partícula.

Se observa que el diferencial de temperatura adquiere un valor positivo para diámetros de partículas mayores a $0,45 \mathrm{~mm}$, no obstante se observa que las diferencias observadas son prácticamente despreciables $\left(<0,05^{\circ} \mathrm{C}\right)$, por lo tanto se considera que la influencia del tamaño de la partícula sobre este factor estudiado es prácticamente nula. Adicionalmente, se considera que el reactor se aproxima a la operación isotérmica.

La variación del rendimiento del hidrógeno generado se presenta en la Figura 9. Se aprecia que a mayores diámetros de partícula, aumenta la generación de hidrogeno en el proceso, debido a que aumenta la conversión global del carbono, por lo cual se infiere que de acuerdo a las restricciones del modelo fluodinámico empleado, que el diámetro óptimo corresponde al mayor evaluado $(0,65 \mathrm{~mm})$. Se puede apreciar que la tendencia es prácticamente lineal, donde el rendimiento tiene un comportamiento creciente en función al aumento del tamaño de la partícula.

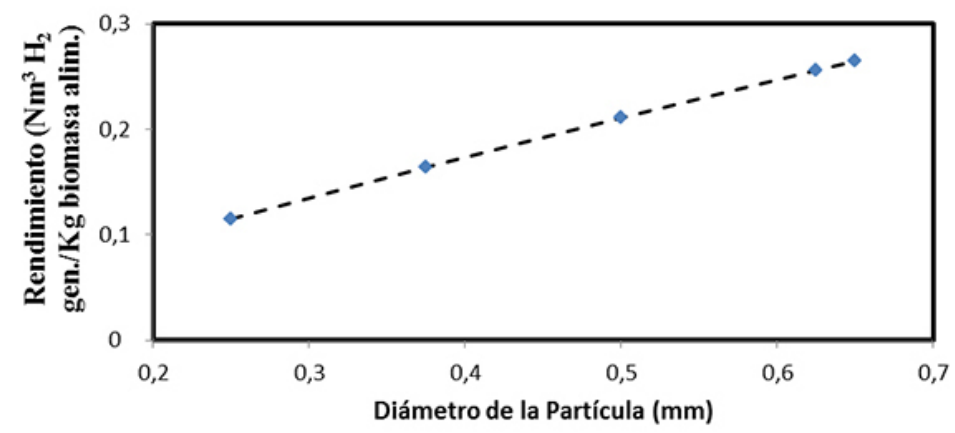

Figura 9. Variación del rendimiento de la reacción de en función del diámetro de la partícula.

La variación de la selectividad instantánea se refleja en la Figura 10. Se observa que la influencia del diámetro de partícula del aserrín alimentado sobre la selectividad de la reacción es realmente significativa, ya que tiende a aumentar exponencialmente al aumentar el diámetro de la partícula dentro del rango estudiado, observándose que crece exponencialmente para el máximo diámetro estudiado. Particularmente, la variación del incremento promedio de la selectividad es de 10,17 por cada aumento de $0,125 \mathrm{~mm}$ de diámetro de partícula de aserrín de pino. Por consiguiente, conviene emplear el máximo diámetro permitido para el modelo fluodinámico, el cual corresponde a $0,65 \mathrm{~mm}$. 


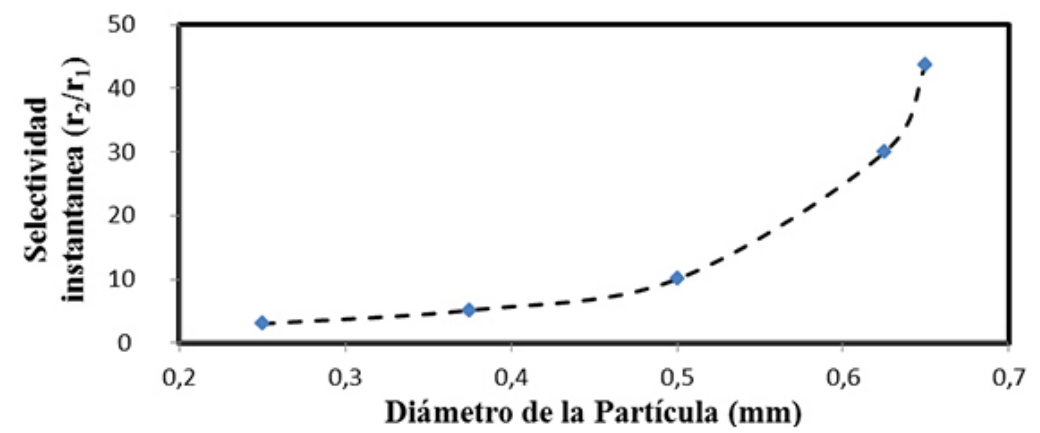

Figura 10. Variación de la selectividad instantánea de la reacción de gasificación en función del diámetro de la partícula.

\section{Caso 3. Variación del radio de vapor de $\mathrm{H}_{2} \mathrm{O} / \mathrm{C}$ alimentado}

En este caso se presentan los resultados y análisis de la variación del radio de vapor de $\mathrm{H}_{2} \mathrm{O} / \mathrm{C}$ alimentado a partir de las condiciones de operación del reactor, tal como se refleja en la Tabla 4.

Tabla 4. Condiciones de operación del reactor.

\begin{tabular}{|l|c|}
\hline \multicolumn{1}{|c|}{ Parámetro } & Valor \\
\hline Flujo biomasa $(\mathrm{kg} / \mathrm{h})$ & 0,444 \\
\hline Flujo aire $\left(\mathrm{Nm}^{3} / \mathrm{h}\right)$ & 0,5 \\
\hline Diámetro de la partícula $(\mathrm{mm})$ & 0,25 \\
\hline Temperatura de entrada $\left({ }^{\circ} \mathrm{C}\right)$ & 900 \\
\hline
\end{tabular}

La variación de la conversión global del carbono se presenta en la siguiente Figura 11. De este resultado se observa que al aumentar el flujo de vapor de agua para una cantidad fija de biomasa procesada (aserrín de pino), la conversión global del carbono en el proceso disminuye, observándose el valor óptimo en 2,375 debido a la inhibición de la degradación de las macromoléculas en la reacción de gasificación para las condiciones de operación del proceso (Durán-García 2014, Guo et al. 2007, Fang et al. 2008, Kumar et al. 2009).

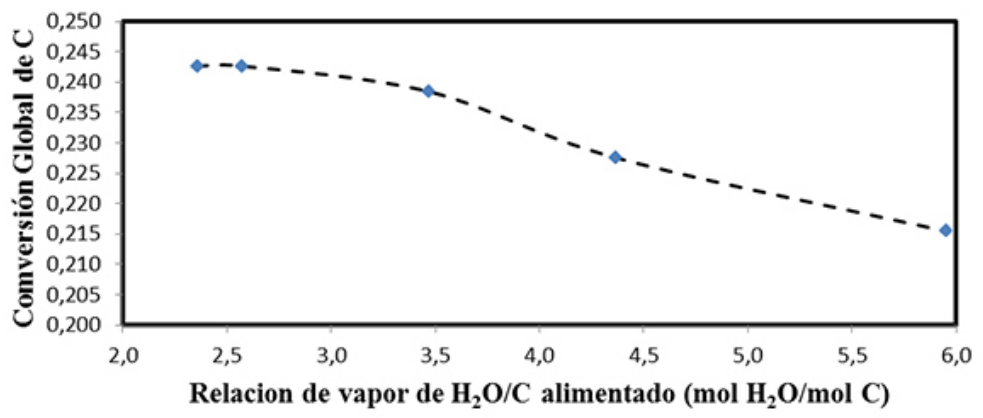

Figura 11. Variación de la conversión global de Carbono en función de la relación de vapor de $\mathrm{H}_{2} \mathrm{O}$ / $\mathrm{C}$ alimentado. 
En cuanto a la variación de la composición del gas de emisión se tiene los siguientes resultados que se puede apreciar en la Figura 12:

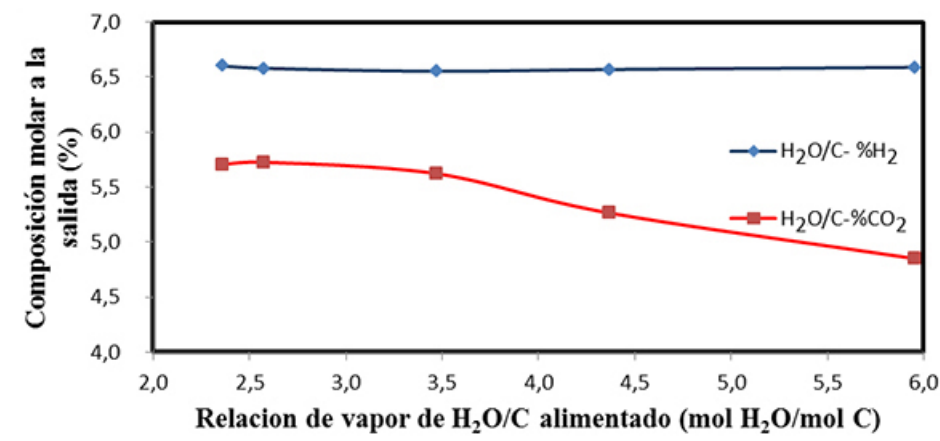

Figura 12. Variación de la composición del hidrógeno y dióxido de carbono en función de la relación de vapor de $\mathrm{H}_{2} \mathrm{O} / \mathrm{C}$ alimentado.

De estos resultados se puede apreciar que la composición molar de hidrógeno prácticamente permanece constante en relación a la variación del radio vapor/biomasa alimentado al proceso de gasificación. Sin embargo, se observa que a medida que aumenta el radio vapor/biomasa alimentado la composición de dióxido de carbono disminuye razonablemente.

En cuanto a la variación de la diferencia de temperatura entre la entrada y salida del reactor, se presenta en la Figura 13:

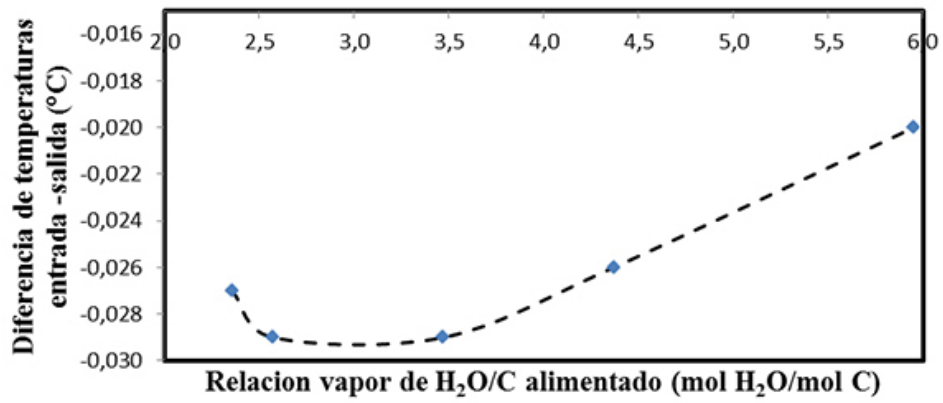

Figura 13. Variación del diferencial de temperatura del reactor en función de la relación vapor $\mathrm{H}_{2} \mathrm{O} / \mathrm{C}$ alimentado.

Se observa que a medida que aumenta el radio vapor de $\mathrm{H}_{2} \mathrm{O} /$ biomasa la tendencia del diferencial es creciente, sin embargo para el rango estudiado las diferencias observadas son poco significativas ya que la variación promedio de las diferencias de temperaturas apenas alcanza los $0,0018^{\circ} \mathrm{C}$ por cada variación de 0,90 de radio vapor de $\mathrm{H}_{2} \mathrm{O}$ /biomasa, por lo que se puede considerar que la influencia de la variación de dicho radio no es suficientemente apreciable.

En cuanto a la variación del rendimiento obtenido se describe su comportamiento en la siguiente Figura 14. Se observa que esta curva tiene un comportamiento análogo al de la conversión global de carbono, donde a mayores conversiones se espera un aumento de la producción de hidrogeno, razón por la cual el máximo rendimiento se presenta para la mínima relación estudiada $(2,375)$. 


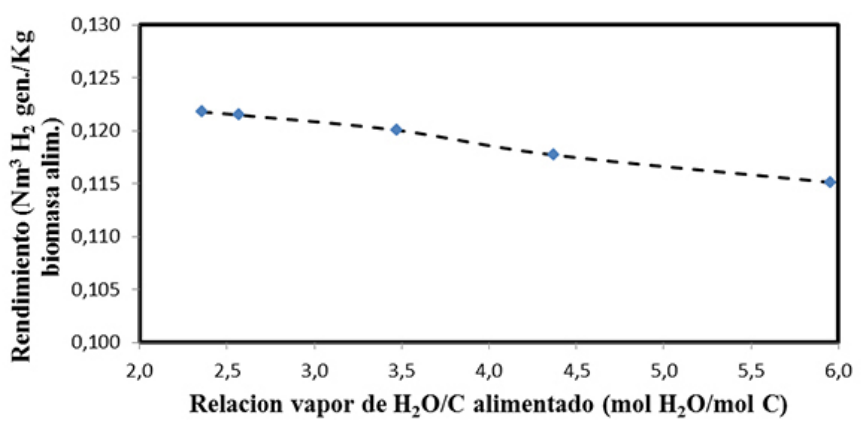

Figura 14. Variación del rendimiento de la reacción en función de la relación vapor $\mathrm{H}_{2} \mathrm{O} / \mathrm{C}$ alimentado.

En cuanto a la variación de la selectividad instantánea se puede apreciar en la Figura 15. Se observa que la selectividad instantánea decrece de manera exponencial a medida que aumenta el radio vapor/biomasa alimentada, de modo que es mucho más beneficioso para el proceso de gasificación bajos radios de los ya mencionados, observándose adicionalmente que para valores menores a 2,5; la selectividad aumenta en una gran proporción, por lo cual se considera operar en el rango de 2,375-2,5 es el mejor rango de operación para esta variable.

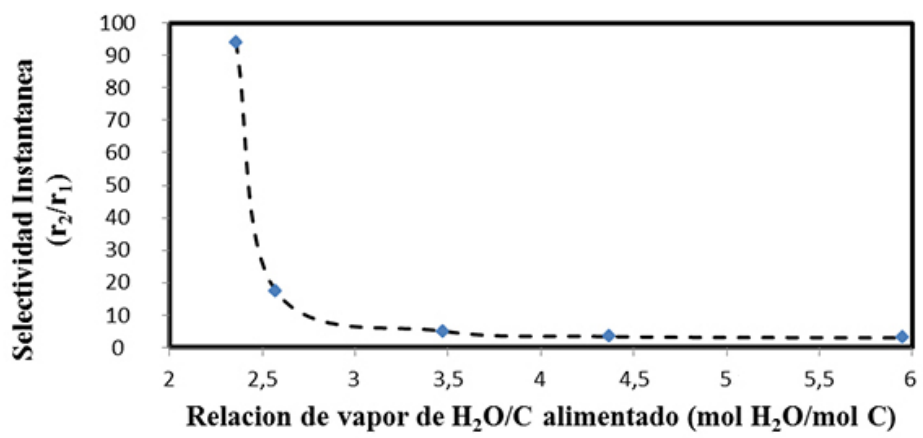

Figura 15. Variación de la selectividad instantánea de la reacción de gasificación en función de la relación de vapor de $\mathrm{H}_{2} \mathrm{O} / \mathrm{C}$ alimentado.

\section{Caso 4. Variación del radio $\mathrm{O}_{2} / \mathrm{C}$ alimentado}

Para estudiar la variación del radio $\mathrm{O}_{2} / \mathrm{C}$ alimentado, se consideran las siguientes condiciones del reactor (Tabla 5).

Tabla 5. Condiciones de operación del reactor.

\begin{tabular}{|l|c|}
\hline \multicolumn{1}{|c|}{ Parámetro } & Valor \\
\hline Flujo biomasa $(\mathrm{kg} / \mathrm{h})$ & 0,444 \\
\hline Flujo de vapor de agua $(\mathrm{kg} / \mathrm{h})$ & 1,8 \\
\hline Diámetro de la partícula $(\mathrm{mm})$ & 0,25 \\
\hline Temperatura de entrada $\left({ }^{\circ} \mathrm{C}\right)$ & 900 \\
\hline
\end{tabular}

Por lo tanto la variación de la conversión global del carbono se presenta en la Figura 16. Se observa 
que la conversión global del carbono aumenta a medida que el flujo de oxigeno es mayor para un volumen fijo de biomasa alimentado, para lo cual de acuerdo a los parámetros operacionales del reactor corresponde a 0,4050. El comportamiento (tendencia) es lineal para este caso.

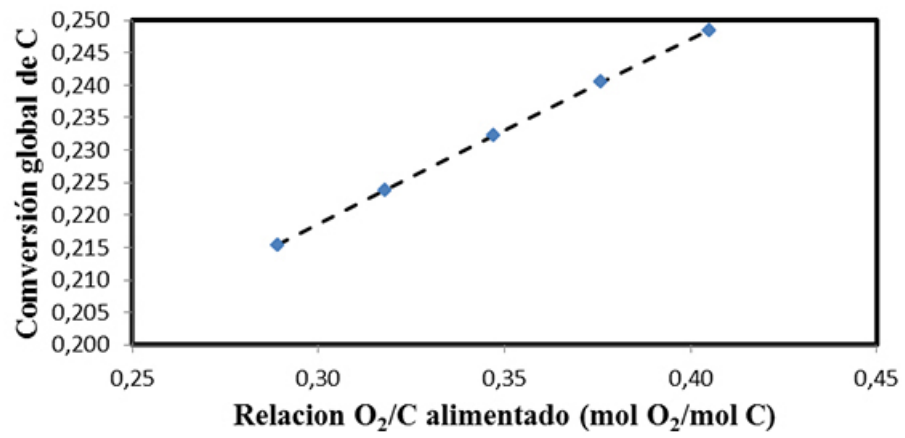

Figura 16. Variación de la conversión global del carbono en función de la relación $\mathrm{O}_{2} / \mathrm{C}$ alimentado.

La variación de la composición del gas de emisión se aprecia en la Figura 17, notándose que a bajos radios de oxigeno/biomasa alimentada la composición de hidrogeno es razonablemente mayor que la dióxido de carbono, y a medida que aumenta la variable estudiada la tendencia es decreciente para la composición de hidrogeno y creciente para de dióxido de carbono, lo cual sugiere que a mayores velocidades de alimentación de oxigeno predomina la reacción de combustión sobre la de gasificación.

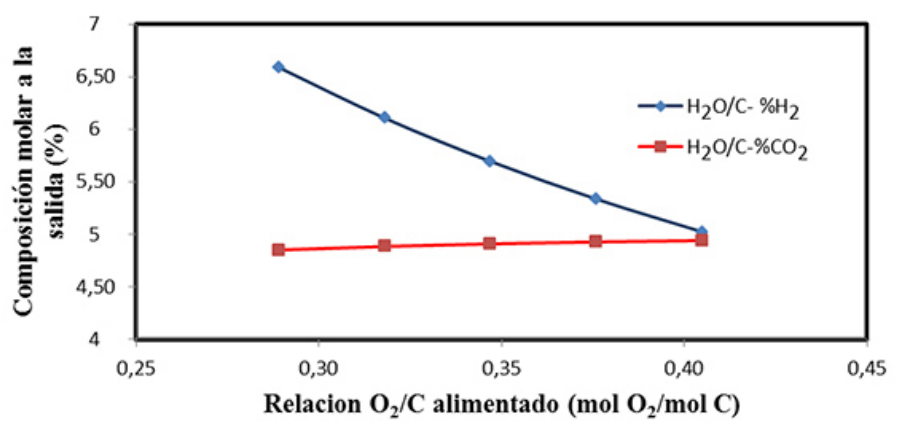

Figura 17. Variación de la composición del hidrógeno y dióxido de carbono en función de la relación $\mathrm{O}_{2} / \mathrm{C}$ alimentado.

La variación del diferencial de temperaturas a la entrada y salida del reactor se muestran en la Figura 18. Se observa una tendencia decreciente, prácticamente de manera lineal al aumentar el radio oxigeno/biomasa alimentada, sin embargo la diferencia es prácticamente mínima entre cada punto evaluado ya que la variación promedio de las diferencias de temperaturas apenas alcanza los $0,0058^{\circ} \mathrm{C}$ por cada variación de 0,0290 de radio vapor de $\mathrm{O}_{2} /$ biomasa, lo que sugiere que la influencia de esta variable no es significativa sobre el diferencial estudiado. 


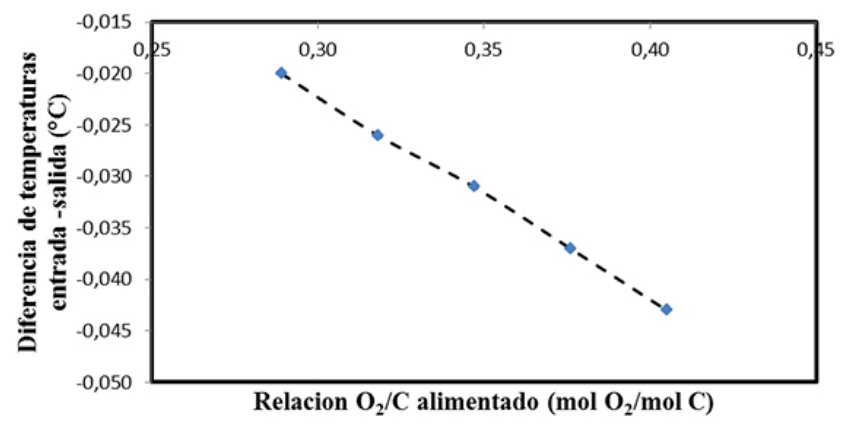

Figura 18. Variación de la composición del gas de emisión en función de la relación vapor $\mathrm{O}_{2} / \mathrm{C}$ alimentado.

La variación del rendimiento obtenido se aprecia en la siguiente Figura 19. En este caso, el rendimiento aumenta de manera aproximadamente lineal creciente en función a la variación de la variable estudiada, sin embargo la variación entre los puntos extremos es inferior al 1\%, por lo tanto la influencia de esta variable es prácticamente despreciable.

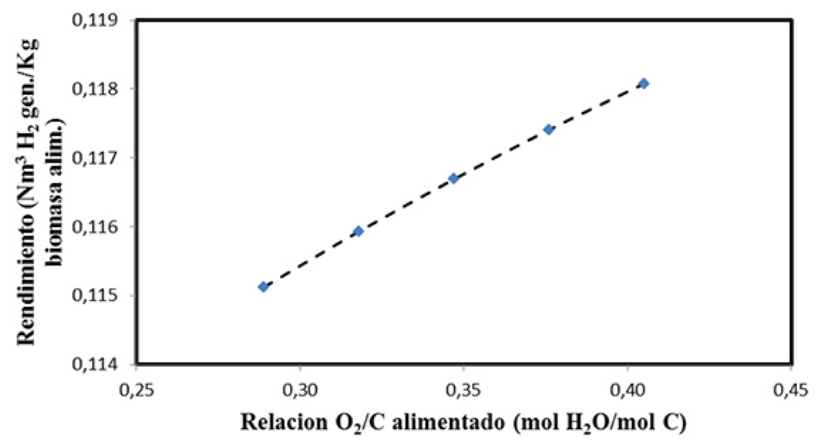

Figura 19. Variación del rendimiento de la reacción en función de la relación vapor $\mathrm{O}_{2} / \mathrm{C}$ alimentado.

La variación de la selectividad instantánea se presenta en la Figura 20. Para este caso se observa una tendencia decreciente a medida que aumenta el flujo de oxígeno al proceso (para un volumen de biomasa constante), lo cual implica que la reacción de gasificación se beneficia a bajos radios de oxigeno alimentados, tomando como referencia el punto de máxima selectividad, el cual correspondió a 0,289 . 


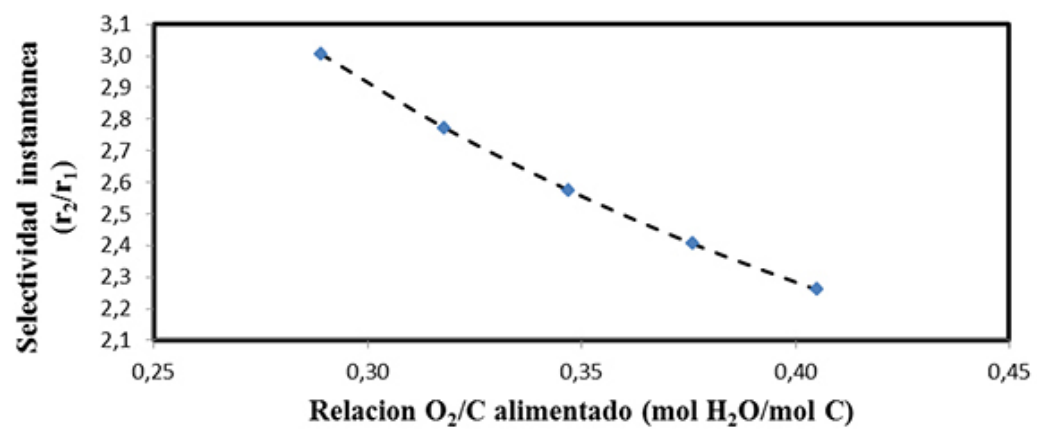

Figura 20. Variación de la selectividad instantánea en función de la relación vapor $\mathrm{O}_{2} / \mathrm{C}$ alimentado.

\section{CONCLUSIONES}

Posterior al análisis de los resultados arrojados por la simulación del sistema de gasificación con aserrín, se pueden establecer las siguientes consideraciones finales:

La reacción de gasificación de aserrín se beneficia a altas temperaturas, por encima de los $900{ }^{\circ} \mathrm{C}$, de manera tal que es requerido operar por encima de ese valor para obtener resultados favorables.

Para todos los casos estudiados, el reactor presentó un comportamiento que tiende a ser isotérmico, por lo cual la energía que requiere es la necesaria para precalentar los reactivos al proceso y poco calor generado en algunos casos (diferenciales de temperatura) resultan muy bajos para sugerir un proceso energéticamente autosostenible.

La reacción se vio beneficiada para tamaños de partículas mayores, observándose el diámetro óptimo para este caso (bajo el modelo fluodinámico planteado) de $0,65 \mathrm{~mm}$.

La reacción se ve favorecida para bajos radios de vapor/biomasa alimentada, observándose como valor óptimo 2,375 .

La reacción de gasificación se ve favorecida para bajos radios aire/biomasa, para lo cual el valor óptimo estudiado fue 0,289 . 


\section{REFERENCIAS}

Antal, M.; Allen, S.; Schulman, D.; Xu, X.; Divilio, R. 2000. Biomass gasification in supercritical water. Ind Eng Chem Res 39:4040-4053.

Beraldo, A.; Balzamo, H. 2009. Compuestos no-estructurales de cementos comerciales y aserrín de maderas argentinas. Maderas-Ciencia y Tecnología 11(3):233-250.

Durán-García, M. E. 2014. Reactores de lecho fluidizado y lecho burbujeante en la gasificación de biomasa residual. Revista Fuentes El Reventón Energético 12:35-43.

Fang, Z.; Minowa, T.; Fang, C.; Smith, R., Inomata, H.; Kozinski, J. 2008. Catalytic hydrothermal gasification of cellulose and glucose. International Journal of Hydrogen Energy 33:981990.

Guo, L.; Lu, Y.; Zhang, X.; Ji, C.; Guan, Y.; Pei, A. 2007. Hydrogen production by biomass gasification in supercritical water: A systematic experimental and analytical study. Catalysis Today 129:275-286.

Kojima, T.; Assavadakorn, P.; Furusawa, T. 1993. Measurement and evaluation of gasification kinetics of sawdust char with steam in an experimental fluidized bed. Fuel Procces Technol 36:201207.

Kumar, A.; Jones, D.; Hanna, M. 2009. Thermochemical Biomass Gasification: A Review of the Current Status of the Technology. Energies 2:556-581.

Kunni, D.; Levenspiel, O. 1991. Fluidization Engineering, $2^{\text {nd }}$ ed. 491p.

Lee, J.; Kim, Y.; Lee, W.; Kim, S. 1998. Coal Gasification Kinetics derived from pirolysis in a fluidized bed reactor. Energy 23:475-488.

Lee, G.; Nunoura, T.; Matsumura, Y.; Yamamoto, K. 2002a. Global kinetics of 2- chlorophenol disappearance with $\mathrm{NaOH}$ in Supercritical Water. J Chemical Engineering of Japan 35:1252-1256.

Lee, I.; Kim, M.; Ihm, S.K. 2002b. Gasification of glucose in supercritical water. Ind Eng Chem Res 41:1182-1188.

Lu, Y.; Guo, L.; Ji, C.; Zhang, X.; Hao, X.; Yan, Q. 2006. Hydrogen production by biomass gasification in supercritical water: A parametric study. International Journal of Hydrogen Energy 31:822-831.

Lu, Y. ; Guo, L. ; Zhang, X. ; Yan, Q. 2007. Thermodynamic modeling and analysis of biomass gasification for hydrogen production in supercritical water. Chemical Engineering Journal 131: 233244.

Lu, Y.; Jin, H.; Guo, L.; Zhang, X.; Cao, C.; Guo, X. 2008. Hydrogen production by biomass gasification in supercritical water with a fluidized bed reactor. International Journal of Hydrogen Energy 33:6066-6075.

Nikoo, M.; Mahinpey, N. 2008. Simulation of biomass gasification in fluidized bed reactor using Aspen Plus. Biomass and Bioenergy 32:1245-1254. 
Ramakers, B.; Riddet, R.; Kerkhop, P. 2004. Fluidization behavior of wood/sand mixtures. Maderas-Ciencia y Tecnología 6:145-153.

Soto, G.; Nuñez, M. 2008. Fabricación de pellets de carbonilla, usando aserrin de pinus radiata (D. Don), como material aglomerante. Maderas-Ciencia y Tecnología 10(2):129-137. 